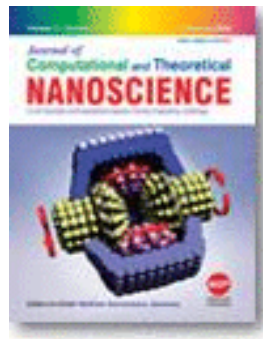

\title{
Preventive and Curative Personality Profiling Based on EEG, ERP, and Big Five Personality Traits: A Literature Review
}

Buy Article:

$\$ 106.46+$ tax

(Refund Policy)

ADD TO CART

BUY NOW

Authors: Saffiera, Cut Amalia; Hassan, Raini; Ismail, Amelia Ritahani

Source: Journal of Computational and Theoretical Nanoscience, Volume 17, Number 2-3, February 2020, pp. 531 545(15)

Publisher: American Scientific Publishers

DOI: https://doi.org/10.1166/jctn.2020.8909

\begin{tabular}{|l|l|l|}
\hline Abstract & $\begin{array}{l}\text { References } \\
\text { Citations }\end{array}$ \\
Supplementary Data Article Media
\end{tabular}

Keywords: Big Five Personality Traits; Curative; Electroencephalography (EEG); Event-Related Potentials (ERP); Healthy Lifestyle; Preventive

Document Type: Research Article

Affiliations: Department of Computer Science, Kuliyyah of Information and Communication Technology, International Islamic University Malaysia, 50728, Kuala Lumpur, Malaysia

Publication date: February 1, 2020

More about this publication? 
